\title{
Cirugía robótica en ginecología: revisión de la literatura
}

\author{
Robotic surgery in gynecology: review of literature
}

\author{
Radamés Rivas-López ${ }^{1 *}$ y Francisco A. Sandoval-García-Travesi ${ }^{2}$

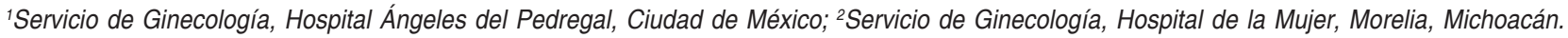 \\ México
}

\section{Resumen}

Antecedentes: La cirugía robótica conlleva el potencial de transformar la cirugía laparoscópica al proporcionar, por primera vez, instrumentos con extremos distales que imitan los intrincados movimientos de la mano humana, mientras que al mismo tiempo proporciona al cirujano una alta definición con visión tridimensional del campo operatorio. Objetivo: Describir la aplicación clínica de la cirugía robótica en padecimientos ginecológicos benignos, así como los componentes del sistema robótico Da Vinci Si, analizando las ventajas y desventajas que este tipo de abordaje quirúrgico brinda a la paciente y a su vez al cirujano. Método: Revisión de la bibliografía en PubMed y UpToDate buscando las palabras clave de nuestra revisión. Criterios de inclusión: artículos de casos o series que contuvieran los apartados de la aplicación de la cirugía robótica en ginecología y resultados clínicos. Resultados: Se encontraron 50 artículos científicos que incluían en sus títulos las palabras clave de nuestra revisión. Se descartaron 22 artículos, 14 por ser inaccesibles, tres debido a que estaban publicados en boletines sin factor de impacto y cinco porque no se obtuvo información sobre los resultados del investigador, su volumen, visibilidad y estructura. Se escogieron para esta revisión 28 artículos. Conclusiones: La cirugía robótica ha evolucionado hasta ser un campo aparte, con un enorme potencial para su futuro desarrollo. Los resultados muestran que esta tecnología es aplicable y capaz de ofrecer un adecuado tratamiento a pacientes seleccionadas.

Palabras Clave: Cirugía ginecológica. Cirugía robótica. Histerectomía robótica. Infertilidad. Miomectomía robótica.

\section{Abstract}

Background: Robotic surgery carries with it the potential to transform laparoscopic surgery by providing, for the $1^{\text {st }}$ time, instruments with distal ends that mimic the intricate movements of the human hand while at the same time providing the surgeon with a high-definition, three-dimensional view of the operative field. Objective: To describe the clinical application of robotic surgery in benign gynecological conditions, as well as the components of the Da Vinci Si robotic system, analyzing the advantages and disadvantages of this type of surgical approach that it provides to the patient and in turn to the surgeon. Method: Review of the literature in PubMed and UpToDate where the keywords of our review were searched. Inclusion criteria: Articles of cases or series containing the sections of the application of robotic surgery in gynecology and clinical results. Results: We found 50 scientific articles that included in their titles the key words of our review. 22 articles were discarded, 14 because they were inaccessible, 3 because they were published in bulletins without an impact factor and 5 because no information was obtained about the researcher's results, their volume, visibility and structure. A total of 28 articles were chosen for this review. Conclusions: Robotic surgery has evolved to be a separate field, with enormous potential for

\footnotetext{
Correspondencia:

*Radamés Rivas-López

Camino Santa Teresa, 1055-129

Col. Héroes de Padierna, Del. Magdalena Contreras

C.P. 10700, Ciudad de México, México

Fecha de recepción: 31-07-2018

Fecha de aceptación: 08-09-2018

E-mail: radamesrl@ hotmail.com

DOI: $10.24875 / C I R U .18000636$ 0009-7411/@ 2018 Academia Mexicana de Cirugía. Publicado por Permanyer. Éste es un artículo open access bajo la licencia CC BY-NC-ND (http://creativecommons.org/licenses/by-nc-nd/4.0/).

Cir Cir. 2020;88(1):107-116 Contents available at PubMed www.cirugiaycirujanos.com
} 
future development. The results show until now that this technology is applicable and capable of offering an adequate treatment to selected patients.

Key Words: Robotic surgery. Gynecological surgery. Robotic hysterectomy. Infertility. Robotic myomectomy.

\section{Introducción}

La cirugía de mínima invasión es punta de lanza en la vanguardia de la tecnología, y la cirugía robótica es parte de este nuevo desarrollo.

En esta revisión de la bibliografía se incluyen las principales cirugías ginecológicas que se benefician ampliamente con el uso de esta tecnología robótica, tales como la histerectomía, la miomectomía, la recanalización tubárica, la sacrocolpopexia, la endometriosis y el cerclaje transabdominal. Así mismo, se analizan las ventajas y desventajas de esta tecnología, así como la influencia del tipo de entrenamiento y de la curva de aprendizaje en este tipo de abordaje quirúrgico.

La cirugía laparoscópica ha evolucionado rápidamente en diversas especialidades. En el área de la ginecología, la cirugía asistida por robot se utiliza en diferentes procedimientos, principalmente en histerectomías por enfermedades benignas yen miomectomías, y también en casos de recanalización tubárica, linfadenectomía, endometriosis y sacrocolpopexia.

La cirugía asistida por robot logró desarrollar avances técnicos que superan a la cirugía laparoscópica, como':

- Visión en tercera dimensión (3D).

- Alta definición visual en la consola.

- Movimientos de las pinzas similares a los de la mano del médico.

- Mejor posición ergonómica durante la cirugía que evita el cansancio.

Los cirujanos que practican la cirugía robótica en padecimientos ginecológicos benignos coinciden en que en algunos casos se reducen la pérdida de sangre y la necesidad de transfusiones, así como el tiempo de estancia hospitalaria y de reintegración a las actividades cotidianas, aunque comúnmente en los primeros casos de cada cirujano el tiempo quirúrgico puede ser más largo que la cirugía laparoscópica, dependiendo de la curva de aprendizaje de cada uno'.

Existen diferentes opiniones en cuanto a las ventajas y desventajas que ofrece la cirugía robótica frente a la cirugía laparoscópica en padecimientos ginecológicos benignos. Por ello, deben conocerse, como en cualquier otro tipo de cirugía, las ventajas de este sistema o abordaje quirúrgico y los riesgos potenciales inherentes a toda cirugía, y notificarlos a la paciente mediante un consentimiento informado antes de la intervención.

Como en cualquier otra técnica quirúrgica, es importante que el cirujano se entrene y certifique conforme a las guías que cada institución hospitalaria indica para la práctica de la cirugía robótica, y que se mantenga actualizado constantemente mediante las herramientas que proporciona la cirugía robótica para asegurarse del uso correcto de esta tecnología y mantener siempre la destreza buscando la seguridad de la paciente en todo momento'.

El objetivo de esta revisión bibliográfica es describir la aplicación clínica de la cirugía robótica en padecimientos ginecológicos benignos.

\section{Método}

Revisión de la bibliografía en las bases de datos de los sitios web de PubMed y UpToDate, en los que se buscaron las palabras clave de nuestra revisión.

\section{Identificación de los estudios}

La búsqueda fue realizada por dos revisores independientes buscando primero los títulos de las palabras clave, y luego se analizaron los resúmenes. No hubo controversias entre los revisores.

\section{Criterios de inclusión}

- Artículos completos en inglés o español desde 1980.

- Artículos de casos o series de casos que contuvieran los apartados de la aplicación de la cirugía robótica en ginecología y resultados clínicos.

\section{Criterios de exclusión}

- Artículos con información incompleta.

- Artículos sin aplicación clínica.

\section{Selección de los estudios}

Se realizaron el análisis y el seguimiento de las publicaciones científicas en revistas indexadas en los 
buscadores de PubMed y UpToDate en el periodo de 1980 a 2018. Después de la identificación de 50 estudios se realizó una revisión bibliométrica para identificar los autores de referencia sobre los temas abordados en nuestra revisión, el lenguaje y la temática, y se descartaron 22 artículos: 14 porque no fue posible su obtención durante la revisión bibliográfica y los ocho restantes porque durante la revisión se identificó que tres estaban publicados en boletines sin factor de impacto y de los cinco restantes no se obtuvo información sobre los resultados del investigador, su volumen, visibilidad ni estructura. Finalmente, se seleccionaron para esta revisión 28 artículos.

\section{Extracción y análisis de los datos}

Durante la investigación, primero se buscaron en inglés los títulos de las palabras clave y posteriormente se analizaron de manera cualitativa los textos de los 28 artículos seleccionados para la revisión, constatando su validez científica y sobre todo su aplicación clínica en nuestro medio.

\section{Resultados}

\section{Antecedentes de la cirugía robótica}

1. Un robot no debe dañar a un ser humano o, por su inacción, dejar que un ser humano sufra daño.

2. Un robot debe obedecer las órdenes dadas por un ser humano, excepto cuando estas órdenes están en oposición con la primera ley.

3. Un robot debe proteger su propia existencia, hasta donde esta protección no esté en conflicto con la primera o segunda leyes.

Lo anterior es un fragmento de la novela futurista de Isaac Asimov Yo, robot, escrita en 1950, que muestra cómo el ser humano siempre ha mantenido un especial interés por el tema robótico'.

En un inicio los robots fueron desarrollados para actuar de manera autónoma por Stanford Research Institute, Department of Defense, The National Aeronautics y Space Administration, para poder atender a los soldados heridos desde un lugar seguro de manera remota. Con el paso del tiempo se dejó de utilizar la tecnología para la telecirugía y se enfocó en mejorar la técnica de cirugía con mínima invasión.

La cirugía asistida por robot surge como una solución para superar los obstáculos que se tienen en la cirugía de mínima invasión, ya que los cirujanos optaban por otras técnicas quirúrgicas al enfrentarse al tiempo que implicaba la curva de aprendizaje, el tiempo quirúrgico que muchas veces implica el procedimiento y el cansancio físico que conlleva al contar con visión bidimensional, y los grados de movimientos limitados y la réplica del temblor de la mano del médico? ${ }^{2}$.

La cirugía robótica utiliza la base de la cirugía laparoscópica complementando con extremos distales que imitan los movimientos de la mano, y además proporciona visión 3D de alta definición, lo que hace que la técnica sea aún menos invasiva al realizar cortes más finos y precisos.

En 2005 se inician los procedimientos en ginecología al ser aprobado por la Food and Drug Administration (FDA). A partir de esa fecha se realizan más de 2000 procedimientos, con un crecimiento del $25 \%$ anual, y los médicos y hospitales impulsan la cirugía robótica y el desarrollo de la tecnología en este ámbito, por lo que surge el Committee on Gynecologic Practice en la Society of Ginecologyc Surgeons (SGS) para regular la información que se brinda a los pacientes, así como para evaluar prácticas, resultados y datos, siempre enfocado a la seguridad del paciente ${ }^{3}$.

El sistema quirúrgico Da Vinci para procedimientos ginecológicos se introdujo en México en el año 2014, siendo el medio privado el principal realizador de esta cirugía hasta el día de hoy. Actualmente México cuenta con robot Da Vinci en cuatro hospitales privados y cuatro hospitales de la red pública del país; hay seis en Ciudad de México, uno en Monterrey y otro en el Estado de México.

\section{Componentes del robot}

Actualmente, la única plataforma aprobada por la FDA y por la Secretaría de Salud en México es el sistema quirúrgico Da Vinci modelo $\mathrm{Si}$, manejado por Intuitive Surgical (Sunnyvale, CA) (Fig. 1).

El sistema quirúrgico robótico $\mathrm{Si}$ consta de los siguientes componentes:

- Un robot que cuenta con tres o cuatro brazos interactivos con trocares de acero inoxidable, más otro brazo que no es manipulado por el robot, sino por el asistente (Fig. 2).

- Una cámara y sistema de visión que permite ver la imagen en 3D de la pelvis, lo que mejora la percepción de la profundidad (Fig. 3).

- Instrumentos que simulan el movimiento de la muñeca (endowrist), es decir, que traducen los movimientos mecánicos del cirujano en tiempo real dentro del paciente (Fig. 3). 


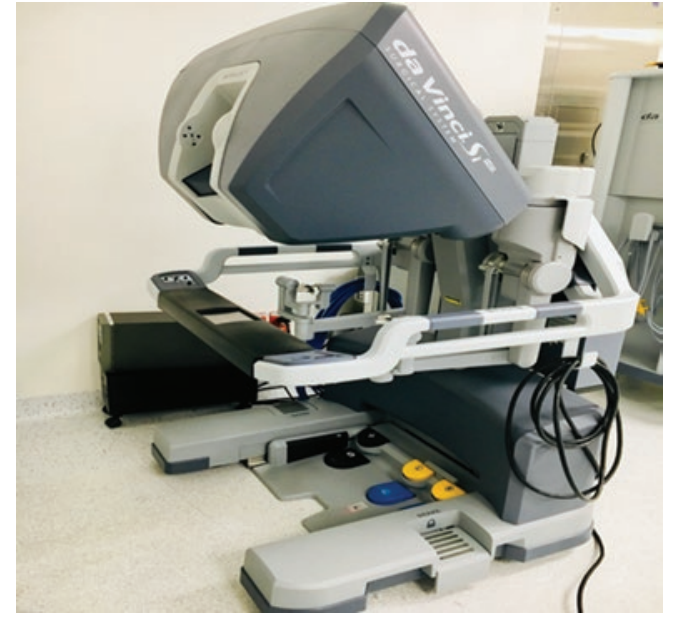

Figura 1. Consola del cirujano.

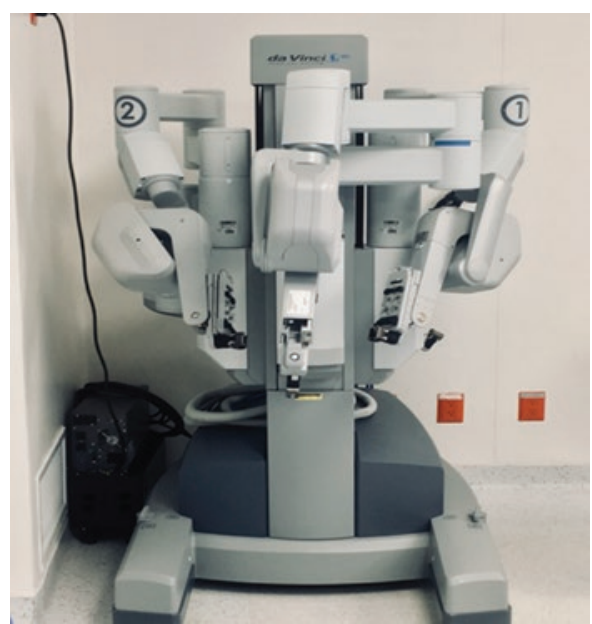

Figura 2. Brazos robóticos.

- Una consola maestra donde el cirujano se sienta frente a la pantalla, al lado del paciente, y desde ahí controla los instrumentos robóticos a través de pinzas que toma con los dedos, y la cámara con la combinación de controles con los pedales $y$ el embrague, con las siguientes funciones (Fig. 4):

- Movimientos: derecha-izquierda, arriba-abajo, entrada-salida.

- Control de la cámara y del enfoque, reposicionamiento de los controles manuales.

- Activación de la fuente de energía monopolar y bipolar.

Durante la cirugía, el médico es capaz de manipular, reposicionar, cortar, diseccionar, coagular y suturar. Aunque el cirujano es prácticamente autónomo, sigue siendo necesario un asistente cuya función es la realización de intercambios, succión e irrigación, así como la introducción de suturas.

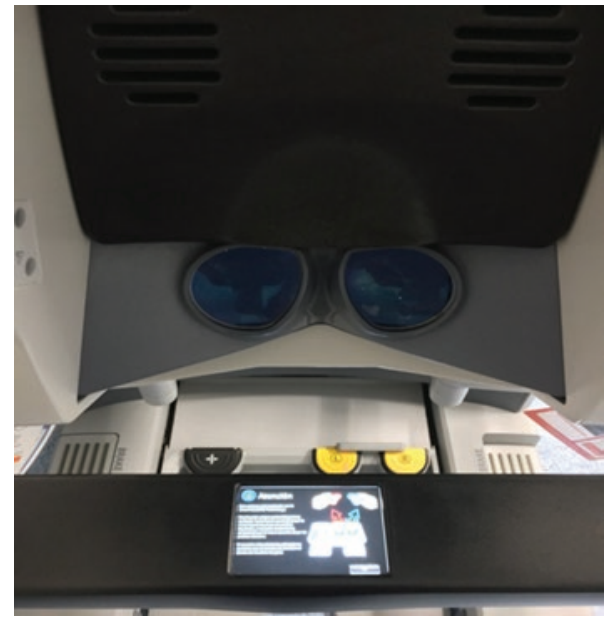

Figura 3. Visor 3D de la consola maestra.

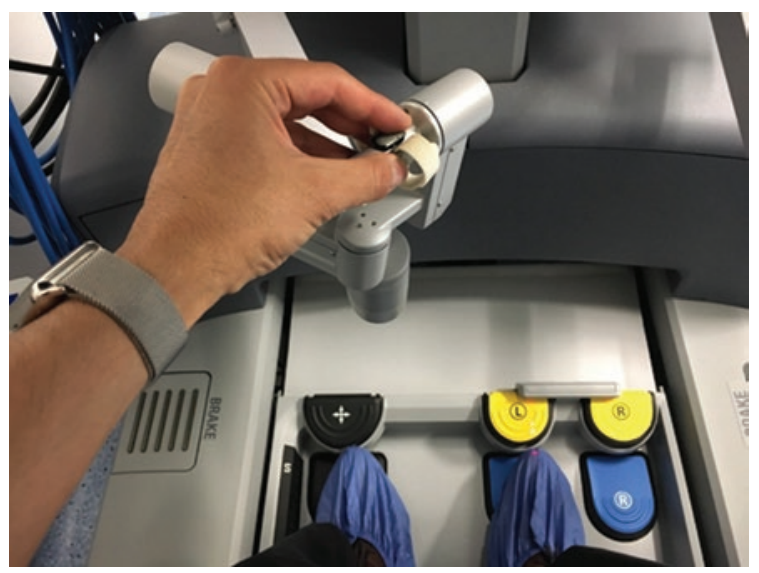

Figura 4. Endowrist y pedales.

\section{Ventajas}

La cirugía robótica ofrece innumerables ventajas sobre la cirugía abierta, por lo que esta no sería un buen punto de comparación. La cirugía robótica debe compararse con la cirugía laparoscópica convencional, y sobre ella tiene varias ventajas:

- La posición ergonómica del médico durante la cirugía, que reduce la fatiga y el malestar. En algunos casos de cirugía laparoscópica, el médico puede tener problemas para manipular los instrumentos y llegar al abdomen de la paciente; en cambio, en la cirugía robótica el cirujano está sentado cómodamente en la consola y manipula los instrumentos mediante las pinzas en sus dedos y los pedales ${ }^{3}$.

- La capacidad del sistema robótico de reducir a cero el efecto del temblor (tremor) del cirujano adicionando precisión y fineza al procedimiento quirúrgico ${ }^{3}$. 
- Instrumentos que simulan la anatomía de la muñeca al contar con siete grados de libertad de movimiento, lo que permite que los movimientos sean naturales y en la dirección que desee el médico. Esto se denomina instrumentación Wristed, que proporciona mayor destreza durante la cirugía (Fig. 4)

- Visión tridimensional y ampliación de las imágenes mediante el ángulo de las cámaras, y alta definición de las imágenes, lo que permite al cirujano contar con una visión exacta e identificar planos tisulares, vasos sanguíneos y nervios. Se ha reportado en estudios comparativos que estas características facilitan que disminuya la pérdida de sangre en las pacientes ${ }^{3}$.

- Mediante la pantalla, el médico puede escribir y dar instrucciones a los ayudantes en cualquier momento de la cirugía. Si se cuenta con un sistema con doble consola, el médico puede señalar y pasar el control al cirujano de la consola vecina para continuar con el procedimiento quirúrgico ${ }^{3}$.

- Habilidad para controlar cuatro instrumentos quirúrgicos, incluida la cámara, lo que permite al cirujano operar con muy poca asistencia por parte del equipo quirúrgico ${ }^{3}$.

\section{Desventajas}

Existen realmente pocas desventajas al utilizar el sistema quirúrgico robótico una vez que se ha logrado alcanzar la curva de aprendizaje. Quizás, haciendo a un lado el costo del equipo en un país en vías de desarrollo, uno de los desafíos para todos los cirujanos es cambiar el paradigma de la falta de tacto en el robot; es decir, al igual que en la cirugía laparoscópica convencional, la ausencia de poder tocar y sentir con las manos cambia por la excepcional visión que se tiene usando la plataforma quirúrgica Da Vinciji-5.

\section{La robótica en procedimientos ginecológicos}

La cirugía robótica en el campo de las enfermedades ginecológicas benignas tiene una amplia indicación. En esta revisión nos enfocaremos exclusivamente en los seis procedimientos principales realizados en patología benigna, que son histerectomía, miomectomía, sacrocolpopexia, reanastomosis tubári$\mathrm{ca}$, endometriosis y cerclaje transabdominal.

\section{Histerectomía}

La histerectomía es el segundo procedimiento ginecológico más común en los EE.UU., con aproximadamente 433,000 casos al año. Se puede realizar de varias maneras: vaginal, por laparoscopía o abdominal. Aunque más de la mitad de los casos se realizan como cirugía abdominal, desde el año 2010 la tendencia es cada vez mayor hacia la cirugía de mínima invasión $n^{5}$. Sin embargo, el presupuesto para la cirugía robótica es limitado y en dos estudios realizados aleatoriamente por la SGS en forma comparativa con la laparoscopía en 148 pacientes, en 2010, el tiempo quirúrgico fue significativamente más largo en la histerectomía asistida por robot (29 minutos con cirugía laparoscópica y 77 minutos con cirugía robótica). El estudio no reportó diferencias en cuanto a pérdida sanguínea, tiempo de estancia hospitalaria, nivel de dolor posoperatorio ni tiempo de recuperación $n^{6,7}$.

En otro estudio realizado de 2007 a 2010 en 264,758 mujeres sometidas a histerectomía con patología benigna, en 441 hospitales, comparando cirugía robótica y cirugía laparoscópica, se registró un tiempo de hospitalización de menos de 2 días (24.9 vs. 19.65\%), aunque el estudio no especifica la estancia media general de las pacientes; sin embargo, el costo aumentó de manera significativa en la cirugía robótica (\$2,189 aproximadamente por cada caso). No se registró ninguna diferencia en cuanto a las tasas de transfusión y las complicaciones hospitalarias ${ }^{8}$.

Un nuevo estudio, usando datos de pacientes de 2009 a 2010, concluyó que la cirugía robótica costaba $\$ 2,489$ más que las histerectomías laparoscópicas, pero las transfusiones disminuyeron y los casos de neumonía posoperatoria también disminuyeron en el grupo de pacientes operadas por cirugía asistida por robot $^{5}$.

Otro estudio comparativo entre histerectomía robótica y cirugía abierta realizado por Silasi, et al. ${ }^{9}$ en 2013 incluyó 60 pacientes, 30 en el grupo de cirugía robótica y 30 en el grupo de laparotomía, con una edad entre 39 y 65 años, un índice de masa corporal en promedio de 30 (obesidad I) y úteros de más de $1 \mathrm{~kg}$ en ambos grupos. Se observó que, si bien en los casos de cirugía robótica se requirió mayor tiempo operatorio, la estancia intrahospitalaria y el sangrado intraoperatorio fueron significativamente menores que con la laparotomía ${ }^{6-9}$.

Un estudio que sí pudo facilitar datos comparativos entre la cirugía robótica y la laparoscópica fue el de Payne y Dauterive ${ }^{10}$, realizado en 2008. Revisaron de 
manera retrospectiva los últimos 200 casos consecutivos. Entre los grupos de pacientes operadas por laparoscopía y por cirugía asistida por robot no se encontraron diferencias significativas en cuanto al tiempo operatorio y el sangrado, aunque el doble de las pacientes operadas por cirugía laparoscópica tuvieron que convertirse a cirugía por laparotomía ${ }^{10}$.

El American College of Obstetricians and Gynecologists y la SGS, después de estudiar los casos desde 2007, recomiendan el registro de los procedimientos asistidos por robot para informar sobre eventos adversos y documentar ventajas y complicaciones de la técnica ${ }^{10}$.

En un análisis con metodología rigurosa que comparó los resultados de 2,300 histerectomías robóticas, 11,952 histerectomías laparoscópicas convencionales, 5,395 histerectomías por vía vaginal y 9,186 histerectomías por laparotomía en casos de patología ginecológica benigna, se llegó a la conclusión de que, en los casos de cirugía robótica cuyos cirujanos y equipo quirúrgico mantengan un alto volumen de intervenciones(mínimo 20 cirugías al año), la cirugía robótica brinda mayores ventajas que el resto de las opciones y de las vías de acceso quirúrgico ${ }^{10-12}$.

Moawad, et al. ${ }^{13}$, en un estudio en el que compararon los pesos de las piezas quirúrgicas (útero) de cirugía robótica y de cirugía laparoscópica convencional, encontraron que el grupo de cirugía laparoscópica tuvo 47 minutos más de cirugía, un costo de $\$ 1,648$ dólares más que el grupo de cirugía robótica y una probabilidad tres veces mayor de que las pacientes tuvieran que reingresar en comparación con el grupo de cirugía robótica. Concluyen que, con un equipo quirúrgico experto de cirugía robótica, la histerectomía para úteros mayores de $750 \mathrm{~g}$ se asocia a menores tiempos quirúrgicos y a un costo hospitalario más bajo $0^{13}$.

Es interesante señalar que en nuestra opinión, así como en varios lugares de los EE.UU., la realización de una histerectomía debido a causas benignas deberá abordarse primariamente por vía vaginal o laparoscópica. En consecuencia, la cirugía robótica la hemos ido reservando para cirugías con mayor grado de dificultad, como los úteros con un aumento de tamaño considerable $(>14 \mathrm{~cm})$ o con importantes distorsiones de su anatomía, casos en los que se sospechen adherencias pélvicas significativas, por ejemplo con endometriosis, miomectomías previas, enfermedad inflamatoria pélvica, abscesos pélvicos o antecedente de embolización de arterias uterinas, entre otros, en los que la disección que se requiere es muy fina y la tecnología robótica brinda excepcionales ventajas, sobre todo en la visualización y el avance en el desempeño operatorio ${ }^{14}$.

\section{Miomectomía}

Con la miomectomía laparoscópica se ha logrado disminuir la morbilidad posoperatoria y, al igual que en la histerectomía, el tiempo de recuperación es más rápido comparado con la laparotomía.

En cuanto a los beneficios que tiene la cirugía robótica, como un tiempo de recuperación más rápido, menos dolor posoperatorio y pronto regreso a las actividades cotidianas en comparación con la laparotomía, el tiempo quirúrgico y el costo hospitalario de la cirugía laparoscópica o la laparotomía en ocasiones pudiera llegar a ser mayor que el de la cirugía robótica. No se ha demostrado que haya menor pérdida de sangre ni que sean menores las complicaciones con la cirugía robótica en comparación con la cirugía laparoscópica.

Sin embargo, en una serie de 872 pacientes, Pitter, et al. ${ }^{14}$ señalaron que la miomectomía múltiple con asistencia robótica tuvo resultados comparables a los de la laparoscopía convencional, con un bajo índice de nacimientos pretérmino $<35$ semanas $(17 \%)$ y reportando una sola rotura uterina $(1 \%)^{14}$.

En nuestro país ha habido una buena aceptación para la utilización de esta plataforma en casos de miomectomías, incluyendo el nacimiento a término de bebes sin el temor de una complicación mayor como lo es la rotura uterina. Esto demuestra que la miomectomía robótica es una vía accesible, eficiente y con resultados halagüeños para pacientes con miomas y que desean un embarazo ${ }^{15,16}$.

Ciertos grupos, con los que estamos totalmente de acuerdo, señalan que la cirugía robótica es una vía quirúrgica efectiva para aquellos casos de mayor complejidad, ya sea por la deformación de la anatomía o por el número de miomas, en los que la ergonomía, el sistema endowrist y la inigualable visión que brinda el robot serían esenciales para el buen desarrollo del caso en particular (Fig. 5) ${ }^{17}$.

\section{REANASTOMOSIS TUBÁRICA}

Las ventajas que tiene la cirugía de mínima invasión, como la precisión de los cortes, el sistema de visión ampliada en 3D y los instrumentos articulados, permiten que la reanastomosis tubárica tenga mejores probabilidades. 


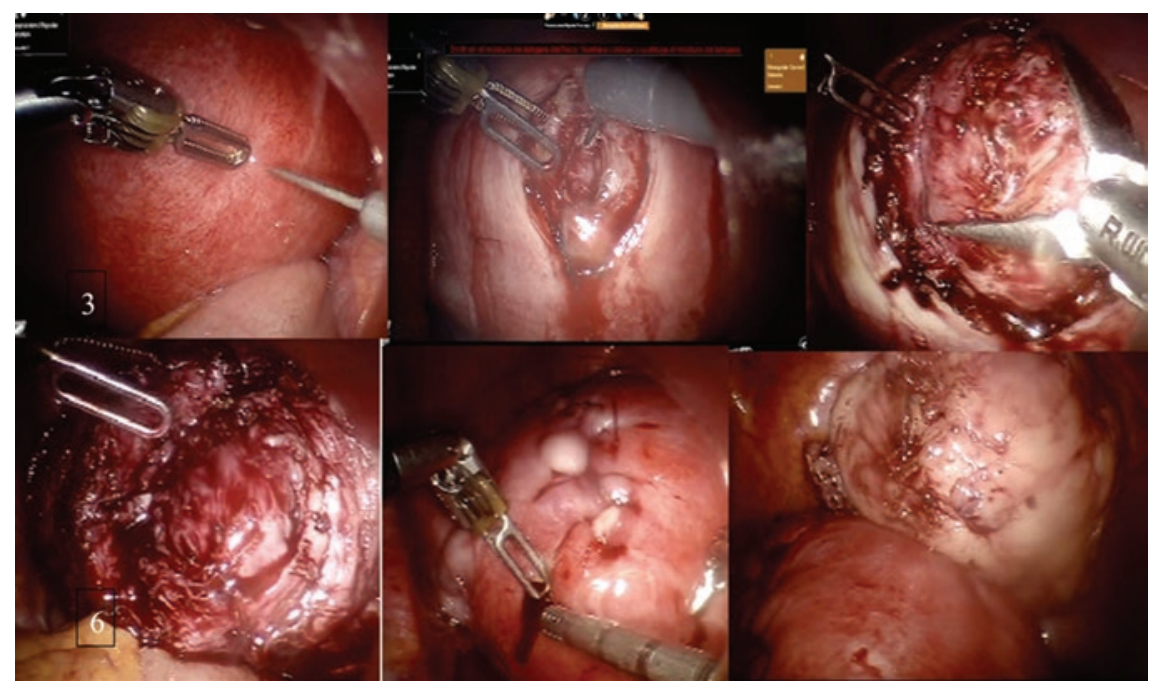

Figura 5. Miomectomía.

En el año 2000, Degueldre, et al..$^{18}$ publicaron un estudio de ocho pacientes con esterilización laparoscópica previa de las trompas para demostrar la factibilidad de la reanastomosis tubárica asistida por robot. Se recanalizaron la totalidad de las trompas en todas las pacientes y el tiempo quirúrgico por cada trompa fue de 52 minutos en promedio. Cinco de las ocho pacientes se sometieron a histerosalpingografía, de las cuales cuatro presentaban permeabilidad bilateral y una unilateral. Hubo dos embarazos reportados dentro de los siguientes 4 meses después de la cirugía. Señalaron que el tiempo comparado con la cirugía convencional fue favorable en la cirugía asistida por robot ${ }^{18}$.

En un segundo estudio con mayor número de pacientes evaluaron la factibilidad de la reanastomosis robótica en comparación con la microcirugía por laparotomía, y encontraron que el tiempo quirúrgico con robot fue mayor (201 frente a 155 minutos con laparotomía). Sin embargo, la estancia hospitalaria fue mucho menor (4 horas con robot frente a 34.7 horas con laparotomía) y el retorno a las actividades cotidianas fue más acelerado (11 días) con la cirugía robótica que con la laparotomía (28 días). Los embarazos logrados también fueron más con la reanastomosis tubárica asistida por robot $(62.5 \%$ frente a $50 \%$ con laparotomía), pero el grupo de pacientes operadas con robot tuvo un número mayor de casos de embarazo ectópico (4 frente a 1 con laparotomía). En cuanto a los costos, no hubo diferencia significativa entre las dos técnicas quirúrgicas. Lo anterior muestra que la reanastomosis tubárica en la era de la fertilización in vitro es un procedimiento plausible para restablecer la fertilidad en pacientes seleccionadas que desean un embarazo posterior a una salpingoclasia bilateral ${ }^{19}$.

\section{SACROCOLPOPEXIA}

El prolapso de la cúpula vaginal es un acontecimiento cada vez más frecuente en la consulta ginecológica. Las opciones quirúrgicas de reconstrucción vaginal, como la fijación del ligamento sacroespinoso, la suspensión del ligamento uterosacro y los procedimientos con malla colocada vaginalmente son tratamientos alternativos, pero tienen diferentes niveles de eficacia y se asocian con riesgos y complicaciones ${ }^{20}$.

La sacrocolpopexia robótica se ha ido desarrollando en los últimos años para tratar este tipo de padecimientos, ya que, en teoría, la realización de esta fijación sería más sencilla que la vía laparoscópica convencional y, por obvias razones, que la cirugía abierta. Geller, et al. ${ }^{21}$ publicaron un estudio retrospectivo 44 meses después de la intervención con 51 pacientes en el que compararon los resultados de la sacrocolpopexia asistida por robot o por laparotomía en el prolapso de órganos pélvicos. Fueron intervenidas con robot 23 pacientes y con laparotomía 28 . Se encontró que entre los dos grupos de pacientes no hubo diferencias significativas en cuanto a mejoría del prolapso, función sexual y función del piso pélvico, pero las intervenidas con robot tuvieron una menor pérdida sanguínea en promedio (151 vs. $219 \mathrm{ml})^{21}$.

Carter, et al. ${ }^{22}$, en otro estudio con seguimiento de 1 año, compararon la sacrocolpopexia robótica y por laparotomía ( $n=17$ y $n=71$, respectivamente) en pacientes con prolapso en estadio III, y encontraron 
tiempos menos prolongados en la cirugía robótica (131 vs. 160 minutos), así como menores tasas de readmisión, recurrencias, días de estancia hospitalaria y complicaciones asociadas. Si se entrena a un equipo quirúrgico en la realización de cirugía robótica y sacrocolpopexia, y solo realiza este tipo de cirugía, los resultados son excelentes en términos de tiempo operatorio, que disminuye casi media hora cuando se compara con el mismo procedimiento con equipos quirúrgicos no exclusivos para el procedimiento ${ }^{22}$.

\section{ENDOMETRIOSIS}

La endometriosis es una enfermedad cada vez más habitual en nuestra sociedad que aqueja a millones de mujeres en todo el mundo, provocando diversos síntomas como dolor pélvico, dismenorrea, inflamación abdominopélvica, infertilidad, etc. El método de referencia para el diagnóstico y el tratamiento de la endometriosis es la cirugía laparoscópica. En los casos de endometriosis profunda, el tratamiento mediante cirugía laparoscópica convencional requiere un desempeño quirúrgico complejo que debe ser realizado en su mayoría por un equipo quirúrgico multidisciplinario. En estos casos, la laparoscopia asistida por robot podría ofrecer ventajas técnicas, como visión 3D, filtración de temblor y mejor ergonomía quirúrgica ${ }^{23}$.

Soto, et al. ${ }^{23}$ realizaron un estudio multicéntrico y aleatorizado que incluyó 73 pacientes (35 en el grupo de cirugía con robot y 38 en el grupo de laparoscopía convencional) intervenidas quirúrgicamente con diagnóstico de endometriosis. Encontraron un tiempo quirúrgico un poco mayor en la cirugía robótica (106 vs. 101 minutos) y no observaron diferencias significativas en cuanto a pérdida sanguínea, complicaciones intraoperatorias o posoperatorias, y tasa de conversión a laparotomía en ambas intervenciones. Además, la mejoría en calidad de vida fue similar en ambos grupos en el seguimiento a 6 meses $^{24}$.

Chen, et al. ${ }^{24}$ realizaron un metaanálisis sobre pacientes intervenidas por endometriosis reuniendo estudios que, al igual que el de Soto, et al. ${ }^{23}$, evaluaron los mismos parámetros, y encontraron un mayor tiempo quirúrgico en el grupo con robot que en el grupo con laparoscopía convencional, sin diferencias significativas en ambos grupos respecto a la pérdida estimada de sangre, la tasa de complicaciones y los días de hospitalización. De este modo, los beneficios de la cirugía robótica en comparación con la laparoscopía convencional en el tratamiento de la endometriosis se sugiere deben seguir estudiándose ${ }^{24}$.

\section{Cerclaje transabdominal}

La insuficiencia cervical se define como una dilatación asintomática del cérvix, que ocurre en el segundo trimestre, principalmente antes de las 24 semanas $^{25}$.

Las indicaciones para la colocación de un cerclaje transabdominal incluyen un cuello uterino corto, ya sea congénito o amputado, una cicatriz cervical que evitaría un abordaje transvaginal y un cerclaje vaginal previo fallido. Hoy en día cada vez existen más reportes sobre la realización de cerclaje transabdominal con la asistencia de la plataforma robótica, teniendo buenos resultados transoperatorios y logrando posteriormente un embarazo a término. Los resultados han sido comparables a los de la cirugía abierta, y los costos muy similares para ambos procedimientos quirúrgicos ${ }^{25}$.

\section{Entrenamiento y curva de aprendizaje}

Con la introducción de la cirugía robótica no se han establecido lineamientos claros que describan cómo debe ser el entrenamiento y en qué debe consistir ${ }^{26}$.

Para el entrenamiento de la cirugía robótica hay varios aspectos similares al de la cirugía laparoscópica; sin embargo, la calidad de las imágenes y los movimientos intuitivos de los instrumentos hacen que sea más ágil el entrenamiento en cirugía robótica. Creemos que es fundamental y de vital trascendencia que, para entrenarse en cirugía robótica, el médico esté capacitado en cirugía laparoscópica ${ }^{26}$.

A diferencia de la cirugía laparoscópica, el médico está más cómodo durante el procedimiento robótico, ya que la posición ergonómica en la que se sienta a un lado de la paciente disminuye la fatiga y el cansancio, lo que permite que se entrene más rápido y pase la curva de aprendizaje en menor tiempo, aunque depende de su destreza y habilidad ${ }^{26}$.

A pesar del rápido crecimiento de la cirugía robótica, en nuestro país no hay muchos cirujanos que se hayan capacitado para practicar esta técnica, por lo que no hay datos contundentes que demuestren cómo debe ser el entrenamiento y no está claro el número de casos que requiere cada cirujano para superar la curva de aprendizaje.

Actualmente, el entrenamiento implica práctica en un quirófano adaptado a la cirugía robótica para que 
el cirujano se familiarice con el entorno, así como con las funciones del robot en cuanto a brazos, trocares y consola. El cirujano, en un inicio, aprende a realizar maniobras simples, ya sea en un cerdo o en tejido humano fresco, tales como el agarre, cortes de diversos tejidos y el nudo intracorpóreo. Se sugiere que el médico practique por lo menos 8 horas en la consola robótica y quede documentado. El entrenamiento debe incluir procedimientos quirúrgicos en cerdos 0 cadáveres humanos ${ }^{3,27}$.

Una vez que el médico concluye el entrenamiento, practicando movimientos en la consola y de forma práctica, tiene que realizar entre dos y cuatro cirugías (dependiendo de los requerimientos de la institución) con la supervisión de un experto o proctor asignado por la institución donde lleve a cabo su práctica (Fig. 5) $)^{3,27}$.

El proctor es un médico certificado en cirugía robótica que ha cumplido con el número de casos que la institución establece y ha superado la curva de aprendizaje. Durante las cirugías se podrá sentar ocasionalmente en la consola para asesorar y aconsejar al cirujano. La pantalla táctil de la torre robótica, en donde se puede escribir y dibujar, es un instrumento útil que se aplica en estos casos ${ }^{3,27}$.

Un estudio retrospectivo evaluó el comportamiento del aprendizaje en la técnica en procedimientos ginecológicos. Se concluyó que los tiempos quirúrgicos se estabilizaron después de 50 casos, pero en un cirujano que realizó 100 histerectomías robóticas se encontró que los tiempos disminuyeron después de los primeros 20 casos, así como las complicaciones clínicas ${ }^{28}$.

Otra ventaja que tiene la plataforma robótica es que, a través de un programa computacional incluido en el software de la consola maestra, el médico puede realizar ejercicios y cirugías de simulación, como un piloto de una aeronave, lo cual brinda grandes ventajas porque mantiene el estar familiarizado con las funciones de la plataforma y ayuda a no perder las habilidades y la coordinación con el sistema robótico ${ }^{28}$.

Existen factores que afectan la curva de aprendizaje, tales como la complejidad de los casos, la experiencia que el médico haya tenido en cirugía laparoscópica y abdominal, y las habilidades y destrezas del equipo quirúrgico. Cada institución tiene un número mínimo de casos que cada médico tiene que cumplir para poder mantener los privilegios para efectuar cirugías asistidas por robot $^{28}$.

Creemos que, al igual que en otros países, a partir de este momento es responsabilidad de los cirujanos, los hospitales y las sociedades médicas determinar el uso más rentable y productivo de esta tecnología.

\section{Conclusiones}

La cirugía de mínima invasión ha tenido un crecimiento exponencial en los últimos años. A medida que la tecnología continúe desarrollándose seguirán evolucionando las técnicas quirúrgicas para ginecología. Es indispensable seguir con la investigación en torno a la cirugía robótica para documentar datos y resultados clínicos que fundamenten las ventajas y los beneficios que puede proporcionar a las pacientes en comparación con la laparoscopía convencional o la cirugía abierta en padecimientos específicos.

\section{Agradecimientos}

Agradecemos a la Revista la aceptación y difusión de esta publicación.

\section{Conflicto de intereses}

Los autores declaran que no hay conflicto de intereses.

\section{Financiamiento}

No se necesitó ningún tipo de inversión económica para esta revisión.

\section{Responsabilidades éticas}

Protección de personas y animales. Los autores declaran que para esta investigación no se han realizado experimentos en seres humanos ni en animales.

Confidencialidad de los datos. Los autores declaran que han seguido los protocolos de su centro de trabajo sobre la publicación de datos de pacientes.

Derecho a la privacidad y consentimiento informado. Los autores han obtenido el consentimiento informado de los pacientes y/o sujetos referidos en el artículo. Este documento obra en poder del autor de correspondencia.

\section{Bibliografía}

1. Asimov I. I, Robot. Greenwich, Conn: Fawcett Publications; 1950.

2. Kwoh YS, Hou J, Jonckheere EA, Hayati S. A robot with improved absolute positioning accuracy for CT guided stereotactic brain surgery. IEEE Trans Biomed Eng. 1988;35:157-60.

3. Visco AG, Advincula AP. Robotic gynecologic surgery. Obstet Gynecol. 2008;112:1369-84. 
4. Truong M, Kim JH, Scheib S, Patzkowsky K. Advantages of robotics in benign gynecologic surgery. Curr Opin Obstet Gynecol. 2016;28:304-10.

5. Alkatout I, Mettler L, Maass N, Ackermann J. Robotic surgery in gynecology. J Turk Ger Gynecol Assoc. 2016;17:224-32.

6. Wright JD, Herzog T, Tsui J, Ananth CV, Lewin SN, Lu YS, et al. Nationwide trends in performance of inpatient hysterectomy in the United States. Obstet Gynecol. 2013;122:233-41.

7. Rosero EB, Kho KA, Joshi GP, Giesecke M, Schaffer Jl. Comparison of robotic and laparoscopic hysterectomy for benign gynecologic disease. Obstet Gynecol. 2013;122:778-86.

8. Online archive of American College of Obstetricians and Gynecologis ACOG Committee Opinion No. 628: Robotic surgery in gynecology. Obstet Gynecol. 2015;125:760-7

9. Silasi DA, Gallo T, Silasi M, Menderes G, Azodi M. Robotic versus abdominal hysterectomy for very large uteri. JSLS. 2013:17:400-6.

10. Olive DL, Parker WH, Cooper JM, Levine RL. The AAGL classification system for laparoscopic hysterectomy (Classification Committee of the American Association of Gynecologic Laparoscopists). J Am Assoc Gynecol Laparosc. 2000;7:9-15.

11. Lim PC, Crane JT, English EJ, Farnam RW, Garza DM, Winter ML, et al Multicenter analysis comparing robotic, open, laparoscopic, and vaginal hysterectomies performed by high-volume surgeons for benign indications. J Gynaecol Obstet. 2016;133:359-64.

12. Moawad GN, Abi Khalil ED, Tyan P, Shu MK, Samuel D, Amdur R, et al. Comparison of cost and operative outcomes of robotic hysterectomy compared to laparoscopic hysterectomy across different uterine weights. J Robot Surg. 2017;11:433-9.

13. Simpson KM, Advincula AP. The essential elements of a robotic-assisted laparoscopic hysterectomy. Obstet Gynecol Clin North Am. 2016;43: 479-93.

14. Pitter MC, Gargiulo AR, Bonaventura LM, Lehman JS, Srouji SS. Pregnancy outcomes following robot-assisted myomectomy. Hum Reprod. 2013:28:99-108.

15. Rivas-López R, Durón-Padilla R, Romero-Hernández S, Audifred-Salomón J, Hernández-Denis JA. Miomectomía laparoscópica asistida po robot y embarazo. Reporte de caso. Ginecol Obstet Mex. 2016;84: 194-200.
16. Kim S, Luu TH, Llarena N, Falcone T. Role of robotic surgery in treating fibroids and benign uterine mass. Best Pract Res Clin Obstet Gynaecol. 2017;45:48-59.

17. Payne TN, Dauterive FR. A comparison of total laparoscopic hysterectomy to robotically assisted hysterectomy: surgical outcomes in a community practice. J Minim Invasive Gynecol. 2008;15:286-91.

18. Degueldre M, Vandromme J, Huong PT, Cadiere GB. Robotically assisted laparoscopic microsurgical tubal reanastomosis: a feasibility study. Fertil Steril. 2000;74:1020-3.

19. Dharia Patel SP, Steinkampf MP, Whitten SJ, Malizia BA. Robotic tubal anastomosis: surgical technique and cost effectiveness. Fertil Steril. 2008;90:1175-9.

20. Daneshgari F, Kefer JC, Moore C, Kaouk J. Robotic abdominal sacrocolpopexy/sacrouteropexy repair of advanced female pelvic organ prolapse (POP): utilizing POP-quantification- based staging and outcomes. BJU Int. 2007;100:875-9.

21. Geller EJ, Parnell BA, Dunivan GC. Robotic vs abdominal sacrocolpopexy: 44-month pelvic floor outcomes. Urology. 2012;79:532-6.

22. Carter-Brooks CM, Du AL, Bonidie MJ, Shepherd JP. The impact of a dedicated robotic team on robotic-assisted sacrocolpopexy outcomes. Female Pelvic Med Reconstr Surg. 2018;24:13-6.

23. Soto $\mathrm{E}$, Luu TH, Liu X, Magrina JF, Wasson MN, Einarsson Jl, et al. Laparoscopy vs. Robotic Surgery for Endometriosis (LAROSE): a multicenter, randomized, controlled trial. Fertil Steril. 2017;107:996-1002.e3.

24. Chen SH, Li ZA, Du XP. Robot-assisted versus conventional laparoscopic surgery in the treatment of advanced stage endometriosis: a meta-analysis. Clin Exp Obstet Gynecol. 2016;43:422-6.

25. Lee R, Biats D, Mancuso M. Robotic transabdominal cerclage: a case series illustrating costs. J Robot Surg. 2018;12:361-4.

26. Carica FA, Vlaovic PD, Chou DS, Abdelshehid CS, White SM, Sala LG, et al. Immediate impact of an intensive one-week laparoscopy training program on laparoscopic skills among postgraduate urologists. J Urol. 2006;175:330.

27. Lenihan JP Jr, Kovanda C, Seshadri-Kreaden U. What is the learning curve for robotic assisted gynecologic surgery? J Minim Invasive Gynecol. 2008;15:589-94

28. Lenihan JP. How to set up a robotic-assisted laparoscopic surgery cente and training of staff. Best Pract Res Clin Obstet Gynaecol. 2017;45:19-31. 